\title{
Differential Games of Mixed Type with Control and Stopping Times
}

\author{
Mrinal K. Ghosh, Mallikarjuna K. S. Rao and Dharmatti Sheetal
}

\begin{abstract}
We study a zero sum differential game of mixed type where each player uses both control and stopping times. Under certain conditions we show that the value function for this problem exists and is the unique viscosity solution of the corresponding variational inequalities. We also show the existence of saddle point equilibrium for a special case of differential game.
\end{abstract}

Mathematics Subject Classification (2000). 90D25, 90D26.

Keywords. Differential game, strategy, stopping time, value, viscosity solution, saddle point equilibrium.

\section{Introduction}

In this article, we study zero-sum differential games of mixed type with both controls and stopping times. Differential games with controls alone have been studied extensively (see [1] and references therein). Stochastic games with stopping times are studied for a class of Markov processes in [6].

For nondegenerate diffusions, the differential games of mixed type have been studied in $[2,5]$. In this case the authors have studied this problem via certain variational inequalities with bilateral constraints. Under a nondegeneracy assumption, they prove the existence of a weak solution of the variational inequalities in certain weighted Sobolev spaces. This result together with certain techniques from stochastic calculus is then applied to get the desired results in stochastic differential games of mixed type. In this paper we study the same problem for a deterministic system. In such a case a classical solution to the corresponding variational inequalities does not exist, even in optimal control problems [1]. We study the problem via the theory of viscosity solutions. We transform the variational inequalities with bilateral constraints to Hamilton-Jacobi-Isaacs equations associated with a differential game problem with control only. Then using standard results from the theory of viscosity solutions, we show that the value function of this differential 
game with control is the unique viscosity solution of the corresponding variational inequalities. Then using penalization method we identify this unique viscosity solution as the value function of the differential game of mixed type. We now describe our problem.

Let $U_{i}, i=1,2$, be compact metric spaces. Let

$$
b: \mathbb{R}^{d} \times U_{1} \times U_{2} \rightarrow \mathbb{R}^{d} .
$$

We assume that

(A1) $b$ is continuous and there exists $C_{1}>0$ such that for all $u_{i} \in U_{i}, i=1,2$,

$$
\left|b\left(x, u_{1}, u_{2}\right)-b\left(y, u_{1}, u_{2}\right)\right| \leq C_{1}|x-y| .
$$

A measurable function $u(\cdot):[0, \infty) \rightarrow U_{i}$ is called an admissible control for the player $i, i=1,2$. Let $\mathcal{A}_{i}, i=1,2$ denote the set of all admissible controls for player $i$. If the players choose controls $\left(u_{1}(\cdot), u_{2}(\cdot)\right) \in \mathcal{A}_{1} \times \mathcal{A}_{2}$, then the state of the system evolves according to

$$
\left.\begin{array}{l}
\dot{x}(t)=b\left(x(t), u_{1}(t), u_{2}(t)\right), \quad t>0 \\
x(0)=x,
\end{array}\right\}
$$

where $x$ is the initial state of system. Under the assumption (A1), (1.1) has a unique (global) solution for all $x \in \mathbb{R}^{d}$. Let

$$
r: \mathbb{R}^{d} \times U_{1} \times U_{2} \rightarrow \mathbb{R}
$$

be the running payoff function and let

$$
\psi_{i}: \mathbb{R}^{d} \rightarrow \mathbb{R}, i=1,2
$$

be the stopping payoffs. We assume that

(A2)(i) $r, \psi_{1}, \psi_{2}$ are continuous, and bounded by $M$.

(ii) There exists a constant $C_{2}>0$ such that for all $x, y \in \mathbb{R}^{d}$ and $u_{i} \in U_{i}$, $i=1,2$

$$
|\eta(x)-\eta(y)| \leq C_{2}|x-y|
$$

for $\eta=r\left(\cdot, u_{1}, u_{2}\right), \psi_{1}, \psi_{2}$.

(iii) $\psi_{2}<\psi_{1}$.

Let $\lambda>0$ be the discount factor. If the players choose controls $\left(u_{1}(\cdot), u_{2}(\cdot)\right) \in$ $\mathcal{A}_{1} \times \mathcal{A}_{2}$ and stopping times $(\theta, \tau) \in \mathbb{R}^{+} \times \mathbb{R}^{+}$, then the total payoff is given by

$$
\begin{aligned}
R\left(x, u_{1}(\cdot), \theta, u_{2}(\cdot), \tau\right)= & \int_{0}^{\theta \wedge \tau} e^{-\lambda t} r\left(x(t), u_{1}(t), u_{2}(t)\right) d t \\
& +e^{-\lambda(\theta \wedge \tau)}\left[\psi_{1}(x(\theta)) \chi_{\theta<\tau}+\psi_{2}(x(\tau)) \chi_{\tau \leq \theta}\right]
\end{aligned}
$$

where $x(\cdot)$ is given by $(1.1)$ under $\left(u_{1}(\cdot), u_{2}(\cdot)\right)$ with initial condition $x(0)=x$.

The aim of player 1 is to minimize the payoff $R\left(x, u_{1}(\cdot), \theta, u_{2}(\cdot), \tau\right)$ over his control and stopping times $\left(u_{1}(\cdot), \theta\right)$, whereas player 2 's objective is to maximize the same over his control and stopping times $\left(u_{2}(\cdot), \tau\right)$.

As is the case with differential games with controls alone, we now introduce the strategies in the sense of Elliott-Kalton. A strategy for the first player is a 
map $\alpha: \mathcal{A}_{2} \rightarrow \mathcal{A}_{1} \times[0, \infty)$ which is nonanticipative in the sense that if for any $t$, $u_{2}(\cdot), \tilde{u}_{2}(\cdot) \in \mathcal{A}_{2}, u_{2}(s)=\tilde{u}_{2}(s)$ for all $0 \leq s<t$, then

$$
\alpha\left[u_{2}\right](s)=\alpha\left[\tilde{u}_{2}\right](s) \text { for all } 0 \leq s<t .
$$

Let $\Gamma$ denote the set of all nonanticipating strategies for player 1 . Similarly nonanticipating strategies for player 2 are defined; the set of all his strategies is denoted by $\Delta$. Let

$$
\begin{aligned}
& V^{+}(x)=\sup _{\tau} \inf _{\theta} \sup _{\beta \in \Delta} \inf _{u_{1} \in \mathcal{A}_{1}} R\left(x, u_{1}(\cdot), \theta, \beta\left(u_{1}(\cdot)\right), \tau\right), \\
& V^{-}(x)=\inf _{\theta} \sup _{\tau} \inf _{\alpha \in \Gamma} \sup _{u_{2} \in \mathcal{A}_{2}} R\left(x, \alpha\left(u_{2}(\cdot), \tau\right), u_{2}(\cdot), \tau\right) .
\end{aligned}
$$

The functions $V^{+}$and $V^{-}$are respectively called upper and lower value functions of the differential game of mixed type. This differential game is said to have value if both upper and lower value functions coincide.

The rest of our paper is structured as follows. In Section 2 we describe the variational inequalities associated with the differential game problem. We establish the existence of a unique viscosity solution (in the class $\mathcal{C}_{b}\left(\mathbb{R}^{d}\right)$ of bounded continous functions) of these inequalities. In Section 3 we establish the existence of value via penalization. As an application, we treat differential game with stopping times alone and show the the existence of a saddle point equilibrium. We also present a direct proof of this result. Section 5 contains some concluding remarks.

\section{Hamilton-Jacobi-Isaacs variational inequalities}

Let $H^{+}, H^{-}: \mathbb{R}^{d} \times \mathbb{R}^{d} \rightarrow \mathbb{R}$ be defined by

$$
\begin{aligned}
& H^{+}(x, p)=\inf _{u_{1} \in U_{1}} \sup _{u_{2} \in U_{2}}\left[b\left(x, u_{1}, u_{2}\right) \cdot p+r\left(x, u_{1}, u_{2}\right)\right], \\
& H^{-}(x, p)=\sup _{u_{2} \in U_{2}} \inf _{u_{1} \in U_{1}}\left[b\left(x, u_{1}, u_{2}\right) \cdot p+r\left(x, u_{1}, u_{2}\right)\right] .
\end{aligned}
$$

Consider the following Hamilton-Jacobi-Isaacs variational inequalities with bilateral constraints

$$
\begin{array}{cl}
\psi_{2}(x) \leq v(x) \leq \psi_{1}(x) & \forall x \\
\lambda v(x)-H^{+}(x, D v(x))=0 & \text { if } \psi_{2}(x)<v(x)<\psi_{1}(x) \\
\lambda v(x)-H^{+}(x, D v(x)) \geq 0 & \text { if } v(x)=\psi_{2}(x) \\
\lambda v(x)-H^{+}(x, D v(x)) \leq 0 & \text { if } v(x)=\psi_{1}(x)
\end{array}
$$

and

$$
\begin{array}{cl}
\psi_{2}(x) \leq v(x) \leq \psi_{1}(x) & \forall x \\
\lambda v(x)-H^{-}(x, D v(x))=0 & \text { if } \psi_{2}(x)<v(x)<\psi_{1}(x) \\
\lambda v(x)-H^{-}(x, D v(x)) \geq 0 & \text { if } v(x)=\psi_{2}(x) \\
\lambda v(x)-H^{-}(x, D v(x)) \leq 0 & \text { if } v(x)=\psi_{1}(x)
\end{array}
$$


By a classical solution of (2.1), we mean a $C^{1}$-function $v$ satisfying (2.1). Similarly a classical solution of $(2.2)$ is defined. We now give an equivalent formulation of these variational inequalities in the following theorem.

Theorem 2.1. Assume (A2)(iii). A function $v \in C^{1}\left(\mathbb{R}^{d}\right)$ is a classical solution of (2.1) if and only if it is a classical solution of the equation

$$
\begin{aligned}
\max \left\{\min \left\{\lambda v(x)-H^{+}(x, D v(x)) ; \lambda\left(v(x)-\psi_{2}(x)\right)\right\} ;\right. & \\
\left.\lambda\left(v(x)-\psi_{1}(x)\right)\right\} & =0 .
\end{aligned}
$$

Similarly a function $v \in C^{1}\left(\mathbb{R}^{d}\right)$ is a classical solution of (2.2) if and only if it is a classical solution of the equation

$$
\begin{aligned}
\min \left\{\max \left\{\lambda v(x)-H^{-}(x, D v(x)) ; \lambda\left(v(x)-\psi_{1}(x)\right)\right\}\right. & \\
\left.\lambda\left(v(x)-\psi_{2}(x)\right)\right\} & =0 .
\end{aligned}
$$

Proof. Let $v$ be a classical solution of (2.1). Suppose $x$ is such that $\psi_{2}(x)<v(x)<$ $\psi_{1}(x)$. Then

$$
\lambda v(x)-H^{+}(x, D v(x))=0, \quad v(x)-\psi_{2}(x)>0, \quad v(x)-\psi_{1}(x)<0 .
$$

Thus (2.3) clearly holds in this case. Now if $v(x)=\psi_{2}(x)$, then

$$
\min \left\{\lambda v(x)-H^{+}(x, D v(x)) ; \lambda\left(v(x)-\psi_{2}(x)\right)\right\}=0,
$$

and hence $(2.3)$ is satisfied. Finally assume $v(x)=\psi_{1}(x)$, then

$$
\min \left\{\lambda v(x)-H^{+}(x, D v(x)) ; \lambda\left(v(x)-\psi_{2}(x)\right)\right\} \leq 0,
$$

and hence

$$
\max \left\{\min \left\{\lambda v(x)-H^{+}(x, D v(x)) ; \lambda\left(v(x)-\psi_{2}(x)\right)\right\} ; \lambda\left(v(x)-\psi_{1}(x)\right)\right\}=0 .
$$

Thus $v$ satisfies (2.3). We now show the converse. It is clear from $(2.3)$ that $v(x) \leq$ $\psi_{1}(x)$. If $v(x)=\psi_{1}(x)$ for some $x$, then it clearly satisfies $v(x) \geq \psi_{2}(x)$ by (A2)(iii). Now let $v(x)<\psi_{1}(x)$. Then from (2.3), we have

$$
\min \left\{\lambda v(x)-H^{+}(x, D v(x)) ; \lambda\left(v(x)-\psi_{2}(x)\right)\right\}=0
$$

and hence $v(x)-\psi_{2}(x) \geq 0$. Thus for all $x$, we have $\psi_{2}(x) \leq v(x) \leq \psi_{1}(x)$. Now let $v(x)<\psi_{1}(x)$. Then from above equation, we have

$$
\lambda v(x)-H^{+}(x, D v(x)) \geq 0 .
$$

Similarly if $v(x)>\psi_{2}(x)$, we can show that

$$
\lambda v(x)-H^{+}(x, D v(x)) \leq 0 .
$$


Thus $v$ is a classical solution of (2.1). This concludes the proof of the first part. The second part of the theorem can be proved in a similar way.

Remark 2.2. Under (A2)(iii), we can also show that a function $v \in C^{1}\left(\mathbb{R}^{d}\right)$ is a classical solution of (2.1) if and only if it is a classical solution of the equation

$$
\min \left\{\max \left\{\lambda v(x)-H^{+}(x, D v(x)) ; \lambda\left(v(x)-\psi_{1}(x)\right)\right\} ; \lambda\left(v(x)-\psi_{2}(x)\right)\right\}=0 .
$$

Similarly a function $v \in C^{1}\left(\mathbb{R}^{d}\right)$ is a classical solution of $(2.2)$ if and only if it is a classical solution of the equation

$$
\max \left\{\min \left\{\lambda v(x)-H^{-}(x, D v(x)) ; \lambda\left(v(x)-\psi_{2}(x)\right)\right\} ; \lambda\left(v(x)-\psi_{1}(x)\right)\right\}=0 \text {. }
$$

Theorem 2.1 motivates us to define viscosity solutions for (2.1) and (2.2) using (2.3) and (2.4) respectively.

Definition 2.3. An upper semicontinuous function $v: \mathbb{R}^{d} \rightarrow \mathbb{R}$ is said to be a viscosity subsolution of (2.1) if it is a viscosity subsolution of (2.3). Similarly a lower semicontinuous function $v: \mathbb{R}^{d} \rightarrow \mathbb{R}$ is said to be a viscosity supersolution of (2.1) if it is a viscosity supersolution of (2.3). A function which is both sub- and supersolution of (2.1) is called a viscosity solution of (2.1). Similarly a viscosity sub-, super- and solutions of (2.2) are defined.

In the classical case, it is quite clear from the proof of Theorem 2.1 that under (A2)(iii), $\psi_{2} \leq v \leq \psi_{1}$ if $v$ is a classical solution of (2.3). We now show that this remains true even for viscosity solutions.

Theorem 2.4. Assume (A2)(iii). Then a viscosity solution of (2.3) satisfies

$$
\psi_{2}(x) \leq v(x) \leq \psi_{1}(x), \text { for all } x \in \mathbb{R}^{d} .
$$

Similarly a viscosity solution of (2.4) satisfies above inequality.

Proof. Let $x \in \mathbb{R}^{d}$. Choose $x_{\epsilon} \in \mathbb{R}^{d}$ such that $x_{\epsilon} \rightarrow x$ and $D^{-} v\left(x_{\epsilon}\right)$ is nonempty (see [1]). Assume $v(x)<\psi_{2}(x)$. Then we can assume that $v\left(x_{\epsilon}\right)<\psi_{2}\left(x_{\epsilon}\right)$. Since $v$ is a viscosity supersolution of $(2.3)$, we have for any $p \in D^{-} v\left(x_{\epsilon}\right)$,

$$
\max \left\{\min \left\{\lambda v\left(x_{\epsilon}\right)-H^{+}\left(x_{\epsilon}, p\right) ; \lambda\left(v\left(x_{\epsilon}\right)-\psi_{2}\left(x_{\epsilon}\right)\right)\right\} ; \lambda\left(v\left(x_{\epsilon}\right)-\psi_{1}\left(x_{\epsilon}\right)\right)\right\} \geq 0 .
$$

Since $v\left(x_{\epsilon}\right)<\psi_{1}\left(x_{\epsilon}\right)$

$$
\min \left\{\lambda v\left(x_{\epsilon}\right)-H^{+}\left(x_{\epsilon}, p\right) ; \lambda\left(v\left(x_{\epsilon}\right)-\psi_{2}\left(x_{\epsilon}\right)\right)\right\} \geq 0 .
$$

Hence $v\left(x_{\epsilon}\right) \geq \psi_{2}\left(x_{\epsilon}\right)$. Now letting $\epsilon \rightarrow 0$, we obtain $v(x) \geq \psi_{2}(x)$ which is a contradiction. Thus $v(x) \geq \psi_{2}(x)$ for all $x \in \mathbb{R}^{d}$. Now assume that there exists $x \in \mathbb{R}^{d}$ such that $\psi_{1}(x)<v(x)$. Again we can find $x_{\epsilon} \in \mathbb{R}^{d}$ such that $x_{\epsilon} \rightarrow x$ 
as $\epsilon \rightarrow 0, D^{+} v\left(x_{\epsilon}\right)$ is nonempty and $v\left(x_{\epsilon}\right)>\psi_{1}\left(x_{\epsilon}\right)$. Now since $v$ is viscosity subsolution, we have for any $p \in D^{+} v\left(x_{\epsilon}\right)$

$$
\max \left\{\min \left\{\lambda v\left(x_{\epsilon}\right)-H^{+}(x, p) ; \lambda\left(v\left(x_{\epsilon}\right)-\psi_{2}\left(x_{\epsilon}\right)\right)\right\} ; \lambda\left(v\left(x_{\epsilon}\right)-\psi_{1}\left(x_{\epsilon}\right)\right)\right\} \leq 0,
$$

which in turn gives

$$
v\left(x_{\epsilon}\right)-\psi_{1}\left(x_{\epsilon}\right) \leq 0 .
$$

Now letting $\epsilon \rightarrow 0$, we obtain $v(x) \leq \psi_{1}(x)$ which is a contradiction. Thus $v(x) \geq$ $\psi_{1}(x)$ for all $x \in \mathbb{R}^{d}$. This completes the proof of the first part of the theorem. The second part can be proved in an analogous way.

We now address the question of showing the existence of unique viscosity solutions of (2.1) and (2.2). This is done by showing that (2.1) and (2.2) are equivalent to Hamilton-Jacobi-Isaacs equations corresponding to a differential game problem with control only. This will achieved by augmenting the control spaces with new controls, we transform the differential game of mixed type to differential game with controls only This method is standard in the literature (see [1], Chapter 3, Section 4.2).

Let $\omega_{1}, \omega_{2}$ be two symbols. We formulate a new differential game problem with control only. In this game, $\bar{U}_{i}$ is the set of controls for player $i$, where $\bar{U}_{i}=$ $U_{i} \cup\left\{\omega_{i}\right\}, i=1,2$. Extend $b, r$ to

$$
\bar{b}: \mathbb{R}^{d} \times \bar{U}_{1} \times \bar{U}_{2} \rightarrow \mathbb{R}^{d}, \quad \bar{r}: \mathbb{R}^{d} \times \bar{U}_{1} \times \bar{U}_{2} \rightarrow \mathbb{R}
$$

respectively such that

$$
\begin{aligned}
\bar{b}\left(x, \omega_{1}, \cdot\right) & \equiv 0, \quad \bar{b}\left(x, \cdot, \omega_{2}\right) \equiv 0, \\
\bar{r}\left(x, \omega_{1}, u_{2}\right) & =\lambda \psi_{1}(x) \forall u_{2} \in U_{2} \quad \text { and } \quad \bar{r}\left(x, \cdot, \omega_{2}\right)=\lambda \psi_{2}(x)
\end{aligned}
$$

The state of the system evolves according to

$$
\left.\begin{array}{l}
\dot{\bar{x}}(t)=\bar{b}\left(\bar{x}(t), \bar{u}_{1}(t), \bar{u}_{2}(t)\right), \quad t>0 \\
\bar{x}(0)=x
\end{array}\right\}
$$

where $\bar{u}_{i}:[0, \infty) \rightarrow \bar{U}_{i}, i=1,2$ are measurable. Let $\overline{\mathcal{A}}_{i}$ denote the set of all measurable functions from $[0, \infty)$ into $\bar{U}_{i}$ for each $i=1,2$. The payoff function is given by

$$
\bar{R}\left(x, \bar{u}_{1}(\cdot), \bar{u}_{2}(\cdot)\right)=\int_{0}^{\infty} e^{-\lambda t} \bar{r}\left(\bar{x}(t), \bar{u}_{1}(t), \bar{u}_{2}(t)\right) d t .
$$

Let $\bar{\Gamma}, \bar{\Delta}$ denote the set of all nonanticipating strategies in this differential game of players 1 and 2 respectively. Then the upper and lower value functions are defined by

$$
\begin{aligned}
& \bar{V}^{+}(x)=\sup _{\beta \in \bar{\Delta}} \inf _{\bar{u}_{1} \in \overline{\mathcal{A}}_{1}} \bar{R}\left(x, \bar{u}_{1}(\cdot), \beta\left[\bar{u}_{1}\right](\cdot)\right) \\
& \bar{V}^{-}(x)=\inf _{\alpha \in \bar{\Gamma}} \sup _{\bar{u}_{2} \in \overline{\mathcal{A}}_{2}} \bar{R}\left(x, \alpha\left[\bar{u}_{2}\right](\cdot), \bar{u}_{2}(\cdot)\right) .
\end{aligned}
$$


Then by standard results [1], under (A1) and (A2), $\bar{V}^{+}$and $\bar{V}^{-}$respectively are unique viscosity solutions of

$$
\lambda v(x)-\bar{H}^{+}(x, D v(x))=0
$$

and

$$
\lambda v(x)-\bar{H}^{-}(x, D v(x))=0,
$$

in the class of bounded continuous functions, where $\bar{H}^{+}, \bar{H}^{-}: \mathbb{R}^{d} \times \mathbb{R}^{d} \rightarrow \mathbb{R}$ are defined as follows:

$$
\begin{aligned}
\bar{H}^{+}(x, p) & =\inf _{\bar{u}_{1} \in \bar{U}_{1}} \sup _{\bar{u}_{2} \in \bar{U}_{2}}\left[\bar{b}\left(x, \bar{u}_{1}, \bar{u}_{2}\right) \cdot p+r\left(x, \bar{u}_{1}, \bar{u}_{2}\right)\right], \\
\bar{H}^{-}(x, p) & =\sup _{\bar{u}_{2} \in \bar{U}_{2}} \sup _{\bar{u}_{1} \in \bar{U}_{1}}\left[\bar{b}\left(x, \bar{u}_{1}, \bar{u}_{2}\right) \cdot p+r\left(x, \bar{u}_{1}, \bar{u}_{2}\right)\right] .
\end{aligned}
$$

We now establish our main theorem of this section which shows the equivalence of (2.1), (2.2) with (2.6), (2.7) respectively.

Theorem 2.5. Assume (A2)(iii). A continuous function $v: \mathbb{R}^{d} \rightarrow \mathbb{R}$ is a viscosity solution of (2.6) if and only if it is a viscosity solution of (2.1). Similarly a continuous function $v: \mathbb{R}^{d} \rightarrow \mathbb{R}$ is a viscosity solution of (2.7) if and only if it is a viscosity solution of $(2.2)$.

Proof. The proof of this theorem is a simple consequence of the observation that

$$
\bar{H}^{+}(x, p)=\left(H^{+}(x, p) \vee \lambda \psi_{2}(x)\right) \wedge \lambda \psi_{1}(x)
$$

and

$$
\bar{H}^{-}(x, p)=\left(H^{-}(x, p) \wedge \lambda \psi_{1}(x)\right) \vee \lambda \psi_{2}(x) .
$$

As a consequence of the above result we have the following existence and uniqueness result for the solutions of (2.1) and (2.2).

Corollary 2.6. Assume (A1) and (A2). Then $\bar{V}^{+}$and $\bar{V}^{-}$are unique viscosity solutions of (2.1) and (2.2) respectively in the class of bounded continuous functions.

Proof. Since (2.6) has a unique viscosity solution in the class of bounded continuous functions given by $V^{+}$, we get by Theorem 2.5 , that $\bar{V}^{+}$is the unique viscosity solution of (2.1) in the class of bounded continuous functions. Similarly $V^{-}$is the unique viscosity solution of (2.2) in the class of bounded continuous functions.

We can also provide comparison principle for the solutions of (2.1) and (2.2). We omit the proof as it is direct from the Theorem 2.5.

Corollary 2.7. Let $v, w$ be sub and super solutions of (2.1) in the class of bounded continuous functions. Then $v \leq w$. Anologous results holds for the solutions of (2.2).

Remark 2.8. In view of the Corollary 2.7, we can now give an alternative proof of Theorem 2.4. The proof depends on the simple observation that that $\psi_{2}$ is a viscosity subsolution of (2.3) and $\psi_{1}$ is a viscosity supersolution of (2.3). 
We say that the differential game problem of mixed type satisfies Isaacs minimax condition if

$$
H^{+}(x, p)=H^{-}(x, p) \text { for all } \quad x, p \in \mathbb{R}^{d} .
$$

The differential game problem with control constructed in this section is said to satisfy Isaacs minimax condition, if for all $x, p \in \mathbb{R}^{d}, \bar{H}^{+}(x, p)=\bar{H}^{-}(x, p)$. We now have the following theorem.

Theorem 2.9. Assume (A1), (A2) and let Isaacs minimax condition hold for the differential game problem of mixed type. Then $\bar{V}^{+} \equiv \bar{V}^{-}$.

Proof. Using (2.8), (2.9) and (A2)(iii), it is easy to verify that $H^{+}(x, p)=H^{-}(x, p)$ if and only if $\bar{H}^{+}(x, p)=\bar{H}^{-}(x, p)$ for all $x, p \in \mathbb{R}^{d}$. Thus the Isaacs minimax condition holds for the differential game problem with control only. Then by the results of Chapter 8 in [1], $\bar{V}^{+} \equiv \bar{V}^{-}$.

Remark 2.10. Let $b\left(x, u_{1}, u_{2}\right)=b_{1}\left(x, u_{1}\right)+b_{2}\left(x, u_{2}\right)$, and $r\left(x, u_{1}, u_{2}\right)=r_{1}\left(x, u_{1}\right)+$ $r_{2}\left(x, u_{2}\right)$, where $b_{i}: \mathbb{R}^{d} \times U_{i} \rightarrow \mathbb{R}^{d}$ and $r_{i}: \mathbb{R}^{d} \times U_{i} \rightarrow \mathbb{R}, i=1,2$. Then Isaacs minimax condition does hold for the differential game problem of mixed type. This condition also holds in the framework of relaxed control. In this framework $U_{i}=$ $\mathcal{P}\left(V_{i}\right), i=1,2$, where $V_{i}$ is a compact metric space and $\mathcal{P}\left(V_{i}\right)$ is the space of probability measures on $V_{i} ; \quad b\left(x, u_{1}, u_{2}\right)=\int_{V_{1}} \int_{V_{2}} \tilde{b}\left(x, v_{1}, v_{2}\right) u_{2}\left(d v_{2}\right) u_{1}\left(d v_{1}\right), r\left(x, u_{1}\right.$, $\left.u_{2}\right)=\int_{V_{1}} \int_{V_{2}} \tilde{r}\left(x, v_{1}, v_{2}\right) u_{2}\left(d v_{2}\right) u_{1}\left(d v_{1}\right)$, for some functions $\tilde{b}: \mathbb{R}^{d} \times V_{1} \times V_{2} \rightarrow \mathbb{R}^{d}$ and $\tilde{r}: \mathbb{R}^{d} \times V_{1} \times V_{2} \rightarrow \mathbb{R}$. Assuming the conditions (A1) and (A2) on $\tilde{b}, \tilde{r}$, we can verify Isaacs minimax condition by using Fan's minimax theorem [4].

\section{Existence of value via penalization}

We assume (A1)-(A2) throughout this section. Consider the following penalized equation

$$
\begin{array}{r}
-\lambda w(x)-\frac{1}{\epsilon}\left(w(x)-\psi_{1}(x)\right)^{+}+\frac{1}{\epsilon}\left(w(x)-\psi_{2}(x)\right)^{-}=H^{-}(x, D w(x)), \\
x \in \mathbb{R}^{d}, \quad \epsilon>0 .
\end{array}
$$

In the following theorem, we prove the existence of viscosity solution to (3.1) and the uniqueness in a suitable class of functions.

Theorem 3.1. There is a unique viscosity solution $w_{\epsilon}$ to $(3.1)$ in $\mathcal{C}_{b}\left(\mathbb{R}^{d}\right)$. Also $w_{\epsilon}$ satisfies the following dynamic programming principle (DPP)

$$
\begin{aligned}
w_{\epsilon}(x)= & \inf _{\alpha \in \Gamma} \sup _{u_{2} \in \mathcal{A}_{2}}\left[\int _ { 0 } ^ { \sigma } e ^ { - \lambda t } \left\{r\left(x(t), \alpha\left[u_{2}\right](t), u_{2}(t)\right)-\frac{1}{\epsilon}\left(w_{\epsilon}(x(t))-\psi_{1}(x(t))\right)^{+}\right.\right. \\
& \left.\left.+\frac{1}{\epsilon}\left(w_{\epsilon}(x(t))-\psi_{2}(x(t))\right)^{-}\right\} d t+w_{\epsilon}(x(\sigma))\right]
\end{aligned}
$$

for any $\sigma \geq 0$. 
Proof. Rewrite the equation (3.1) as follows:

$$
\begin{aligned}
-\left(\lambda+\frac{2}{\epsilon}\right) w_{\epsilon}(x)+w_{\epsilon}(x) \wedge \psi_{1}(x)+w_{\epsilon}(x) & \vee \psi_{2}(x) \\
& =H^{-}\left(x, D w_{\epsilon}(x)\right), \quad x \in \mathbb{R}^{d} .
\end{aligned}
$$

Now we can invoke the standard arguments (see [1]) to prove the existence of a unique viscosity solution in $\mathcal{C}_{b}\left(\mathbb{R}^{d}\right)$. Following Chapter VII, [1], we can prove the DPP.

We now provide a representation formula which is useful in proving the convergence of $w_{\epsilon}$ as $\epsilon$ goes to zero. To this end, let $\mathcal{N}_{\epsilon}$ denote the set of all nonnegative measurable functions $\nu: \mathbb{R} \rightarrow \mathbb{R}$ such that there is a sequence $\left\{\nu_{n}\right\} \subset \mathcal{C}_{+}$ such that $\nu_{n} \rightarrow \nu$ pointwise and $\nu_{n} \leq \frac{1}{\epsilon}$, where $\mathcal{C}_{+}$denotes the set of non-negative continuous functions on $\mathbb{R}$. Set

$$
\begin{aligned}
\tilde{R}\left(x, u_{1}(\cdot), \nu_{1}(\cdot), u_{2}(\cdot), \nu_{2}(\cdot)\right)= & \int_{0}^{\infty} e^{\left(-\int_{0}^{t}\left(\lambda+\nu_{1}(s)+\nu_{2}(s)\right) d s\right)}\left\{r\left(x(t), u_{1}(t), u_{2}(t)\right)\right. \\
& \left.+\nu_{2}(t) \psi_{2}(x(t))+\nu_{1}(t) \psi_{1}(x(t))\right\} d t
\end{aligned}
$$

for $\nu_{1}, \nu_{2} \in \mathcal{N}_{\epsilon}, u_{i} \in \mathcal{A}_{i}$ and $x \in \mathbb{R}^{d}$.

Lemma 3.2. The unique viscosity solution $w_{\epsilon}$ of $(3.1)$ in $\mathcal{C}_{b}\left(\mathbb{R}^{d}\right)$ is given by

$$
\begin{aligned}
w_{\epsilon}(x) & =\sup _{\nu_{2}} \inf _{\nu_{1}} \inf _{\alpha \in \Gamma} \sup _{u_{2} \in \mathcal{A}_{2}} \tilde{R}\left(x, \alpha\left[u_{2}\right](\cdot), \nu_{1}(\cdot), u_{2}(\cdot), \nu_{2}(\cdot)\right) \\
& =\inf _{\nu_{1}} \sup _{\nu_{2}} \inf _{\alpha \in \Gamma} \sup _{u_{2} \in \mathcal{A}_{2}} \tilde{R}\left(x, \alpha\left[u_{2}\right](\cdot), \nu_{1}(\cdot), u_{2}(\cdot), \nu_{2}(\cdot)\right) \\
& =\inf _{\alpha \in \Gamma} \sup _{u_{2} \in \mathcal{A}_{2}} \tilde{R}\left(x, \alpha\left[u_{2}\right](\cdot), \hat{\nu}_{1}(\cdot), u_{2}(\cdot), \hat{\nu}_{2}(\cdot)\right)
\end{aligned}
$$

where $\hat{\nu}_{1}(t)=\frac{1}{\epsilon} \chi_{\left\{w_{\epsilon}(x(t))>\psi_{2}(x(t))\right\}}$ and $\hat{\nu}_{2}(t)=\frac{1}{\epsilon} \chi_{\left\{w_{\epsilon}(x(t))<\psi_{1}(x(t))\right\}}$.

Proof. Observe that

$$
\begin{aligned}
w_{\epsilon}(x)= & \inf _{\alpha \in \Gamma} \sup _{u_{2} \in \mathcal{A}_{2}}\left[\int _ { 0 } ^ { \infty } e ^ { - \int _ { 0 } ^ { t } ( \lambda + \nu ( s ) ) d s } \left\{r\left(x(t), \alpha\left[u_{2}\right](t), u_{2}(t)\right)\right.\right. \\
& -\frac{1}{\epsilon}\left(w_{\epsilon}(x(t))-\psi_{1}(x(t))\right)^{+}+\frac{1}{\epsilon}\left(w_{\epsilon}(x(t))-\psi_{2}(x(t))\right)^{-} \\
& \left.\left.+\nu(t) w_{\epsilon}(x(t))\right\} d t\right]
\end{aligned}
$$


for any $\nu \in \mathcal{C}_{+}$and hence for any $\nu \in \mathcal{N}_{\epsilon}$. Then for any $\nu_{1}, \nu_{2} \in \mathcal{N}_{\epsilon}$, we have

$$
\begin{aligned}
w_{\epsilon}(x)=\inf _{\alpha \in \Gamma} & \sup _{u_{2} \in \mathcal{A}_{2}} \int_{0}^{\infty} e^{-\int_{0}^{t}\left(\lambda+\nu_{1}(s)+\nu_{2}(s)\right) d s}\left\{r\left(x(t), \alpha\left[u_{2}\right](t), u_{2}(t)\right)\right. \\
& +\nu_{2}(t) \psi_{2}(x(t))+\nu_{1}(t) \psi_{1}(x(t))+\nu_{1}(t)\left(w_{\epsilon}(x(t))-\psi_{1}(x(t))\right) \\
& -\frac{1}{\epsilon}\left(w_{\epsilon}(x(t))-\psi_{1}(x(t))\right)^{+} \\
& \left.+\nu_{2}(t)\left(w_{\epsilon}(x(t))-\psi_{2}(x(t))\right)-\frac{1}{\epsilon}\left(w(x(t))-\psi_{2}(x(t))\right)^{-}\right\} d t .
\end{aligned}
$$

Note that

$$
\nu_{1}(t)\left(w_{\epsilon}(x(t))-\psi_{1}(x(t))\right)-\frac{1}{\epsilon}\left(w_{\epsilon}(x(t))-\psi_{1}(x(t))\right)^{+} \leq 0
$$

and

$$
\nu_{2}(t)\left(w_{\epsilon}(x(t))-\psi_{2}(x(t))\right)-\frac{1}{\epsilon}\left(w_{\epsilon}(x(t))-\psi_{2}(x(t))\right)^{-} \geq 0
$$

since $\nu_{1}, \nu_{2} \in \mathcal{N}_{\epsilon}$ and the equality holds true for $\nu_{i}=\hat{\nu}_{i}$ for $i=, 1,2$. Thus it follows that

$$
\begin{aligned}
w_{\epsilon}(x) \geq & \inf _{\alpha \in \Gamma} \sup _{u_{2} \in \mathcal{A}_{2}} \int_{0}^{\infty} e^{-\int_{0}^{t}\left(\lambda+\nu_{1}(s)+\nu_{2}(s)\right) d s}\left\{r\left(x(t), \alpha\left[u_{2}\right](t), u_{2}(t)\right)\right. \\
& +\nu_{2}(t) \psi_{2}(x(t))+\nu_{1}(t) \psi_{1}(x(t))+\left(\nu_{1}(t)\left(w_{\epsilon}(x(t))-\psi_{1}(x(t))\right)\right. \\
& \left.\left.-\frac{1}{\epsilon}\left(w_{\epsilon}(x(t))-\psi_{1}(x(t))\right)^{+}\right)\right\} d t
\end{aligned}
$$

and

$$
\begin{aligned}
w_{\epsilon}(x) \leq & \inf _{\alpha \in \Gamma} \sup _{u_{2} \in \mathcal{A}_{2}} \int_{0}^{\infty} e^{-\int_{0}^{t}\left(\lambda+\nu_{1}(s)+\nu_{2}(s)\right) d s}\left\{r\left(x(t), \alpha\left[u_{2}\right](t), u_{2}(t)\right)\right. \\
& +\nu_{2}(t) \psi_{2}(x(t))+\nu_{1}(t) \psi_{1}(x(t))+\left(\nu _ { 2 } ( t ) \left(w_{\epsilon}(x(t))\right.\right. \\
& \left.\left.\left.-\psi_{2}(x(t))\right)-\frac{1}{\epsilon}\left(w(x(t))-\psi_{2}(x(t))\right)^{-}\right)\right\} d t
\end{aligned}
$$

for any $\nu_{1}$ and $\nu_{2}$. Also the inequalities become equalities when $\nu_{i}=\hat{\nu}_{i}, i=1,2$. Using these inequalities, the desiring result follows.

We next derive some estimates on $w_{\epsilon}$.

Lemma 3.3. There is a constant $C>0$ depending on $b, r, \psi_{1}$ and $\psi_{2}$ such that

$$
\left(w_{\epsilon}-\psi_{1}(x)\right)^{+} \leq C \epsilon \quad \text { and } \quad\left(w_{\epsilon}-\psi_{2}(x)\right)^{-} \leq C \epsilon .
$$


Proof. For any $\alpha \in \Gamma, u_{2}(\cdot) \in \mathcal{A}_{2}$ and non-negative measurable functions $\nu_{1}, \nu_{2}$, we have using integrations by parts

$$
\begin{aligned}
\psi_{1}(x)= & -\int_{0}^{\infty} e^{-\int_{0}^{t}\left(\lambda+\nu_{1}(s)+\nu_{2}(s)\right) d s}\left\{b\left(x(s), \alpha\left[u_{2}\right](s), u_{2}(s)\right) \cdot D \psi_{1}(x(s))\right. \\
& \left.-\lambda \psi_{1}(x(s))-\psi_{1}(x(s))\left(\nu_{1}(s)+\nu_{2}(s)\right)\right\} d s .
\end{aligned}
$$

Hence from Lemma 3.2, we have

$$
\begin{aligned}
w_{\epsilon}(x)- & \psi_{1}(x) \\
= & \inf _{\nu_{1}} \sup _{\nu_{2}} \inf _{\alpha \in \Gamma} \sup _{u_{2} \in \mathcal{A}_{2}} \int_{0}^{\infty} e^{-\left(\lambda t+\int_{0}^{t}\left(\nu_{1}(s)+\nu_{2}(s)\right) d s\right)}\left\{r\left(x(t), \alpha\left[u_{2}\right](t), u_{2}(t)\right)\right. \\
& +b\left(x(t), \alpha\left[u_{2}\right](t), u_{2}(t)\right) \cdot D \psi_{1}(x(t))-\lambda \psi_{1}(x(t)) \\
& \left.+\nu_{2}(s)\left\{\psi_{2}(x(s))-\psi_{1}(x(s))\right\}\right\} d t .
\end{aligned}
$$

Thus for $\nu_{1}=\frac{1}{\epsilon}$, we have

$$
\begin{gathered}
w_{\epsilon}(x)-\psi_{1}(x) \leq \sup _{\nu_{2}} \inf _{\alpha \in \Gamma} \sup _{u_{2} \in \mathcal{A}_{2}} \int_{0}^{\infty} e^{-\left(\lambda t+\frac{1}{\epsilon} t+\int_{0}^{t} \nu_{2}(s) d s\right)}\left\{r\left(x(t), \alpha\left[u_{2}\right](t), u_{2}(t)\right)\right. \\
\left.+b\left(x(t), \alpha\left[u_{2}\right](t), u_{2}(t)\right) . D \psi_{1}(x(t))-\lambda \psi_{1}(x(t))\right\} d t .
\end{gathered}
$$

Now using the bounds of $b, r, \psi_{1}$, we can find $C>0$ such that

$$
\left|r\left(x(t), \alpha\left[u_{2}\right](t), u_{2}(t)\right)+b\left(x(t), \alpha\left[u_{2}\right](t), u_{2}(t)\right) \cdot D \psi_{1}(x(t))-\lambda \psi_{1}(x(t))\right| \leq C .
$$

Also

$$
e^{-\left(\lambda t+\frac{1}{\epsilon} t \int_{0}^{t} \nu_{2}(s) d s\right)} \leq e^{\lambda t+\frac{1}{\epsilon} t}
$$

for any $\nu_{2}$. Now using these bounds, we obtain

$$
w_{\epsilon}(x)-\psi_{1}(x) \leq C \frac{\epsilon}{\lambda \epsilon+1}
$$

for some positive constant $C$. Thus

$$
\left(w_{\epsilon}-\psi_{1}(x)\right)^{+} \leq C \epsilon .
$$

In an analogous way we can prove

$$
\left(w_{\epsilon}-\psi_{2}(x)\right)^{-} \leq C \epsilon .
$$

This completes the proof of the lemma.

We are now ready to prove the convergence of $w_{\epsilon}(\cdot)$ to a continuous function $v(\cdot)$ where $v(\cdot)$ is a viscosity solution of the variational inequalitiy (2.2). This is the content of the following theorem. We adapt Barles-Perthame procedure to prove this theorem (see $[1$, Chapter V]).

Theorem 3.4. The unique viscosity solution $w_{\epsilon}(\cdot)$ to $(3.1)$ in $\mathcal{C}_{b}\left(\mathbb{R}^{d}\right)$ converges to a continuous function $v(\cdot)$ in $\mathcal{C}_{b}\left(\mathbb{R}^{d}\right)$ as $\epsilon \rightarrow 0$ and $v(\cdot)$ is the unique viscosity solution in $\mathcal{C}_{b}\left(\mathbb{R}^{d}\right)$ of the variational inequality $(2.2)$. 
Proof. Lemma 3.3 guarantees that $w_{\epsilon}$ is uniformly bounded. Thus the half-limits

$$
\begin{aligned}
& v_{-}(x)=\liminf _{(y, \epsilon) \rightarrow\left(x, o^{+}\right)} w_{\epsilon}(y) \\
& v^{+}(x)=\limsup _{(y, \epsilon) \rightarrow\left(x, o^{+}\right)} w_{\epsilon}(y)
\end{aligned}
$$

are well-defined. We also have

$$
\psi_{2} \leq v_{-}, \quad v^{+} \leq \psi_{1} .
$$

We now prove that $v^{+}$is a viscosity subsolution of the variational inequality (2.2). Suppose $\psi_{2}(\bar{x})<v^{+}(\bar{x})$ and $v^{+}-\phi$ has a strict local maximum at $\bar{x}$ for a smooth function $\phi$. Choose $x_{\epsilon}$ such that $w_{\epsilon}-\phi$ has a local maximum at $x_{\epsilon}$ and $x_{\epsilon} \rightarrow \bar{x}$ as $\epsilon \rightarrow 0$. Therefore

$$
\lambda w_{\epsilon}\left(x_{\epsilon}\right)-\frac{1}{\epsilon}\left(w_{\epsilon}\left(x_{\epsilon}\right)-\psi_{1}\left(x_{\epsilon}\right)\right)^{+}+\frac{1}{\epsilon}\left(w_{\epsilon}\left(x_{\epsilon}\right)-\psi_{2}\left(x_{\epsilon}\right)\right)^{-} \leq H^{-}\left(x, D \phi\left(x_{\epsilon}\right)\right) .
$$

Multiplying both sides by $\left(w_{\epsilon}\left(x_{\epsilon}\right)-\psi_{1}\left(x_{\epsilon}\right)\right)^{-}$and noting the facts that

$$
\frac{1}{\epsilon}\left(w_{\epsilon}\left(x_{\epsilon}\right)-\psi_{1}\left(x_{\epsilon}\right)\right)^{+}\left(w_{\epsilon}\left(x_{\epsilon}\right)-\psi_{1}\left(x_{\epsilon}\right)\right)^{-}=0
$$

and

we obtain

$$
\frac{1}{\epsilon}\left(w_{\epsilon}\left(x_{\epsilon}\right)-\psi_{2}\left(x_{\epsilon}\right)\right)^{-}\left(w_{\epsilon}\left(x_{\epsilon}\right)-\psi_{1}\left(x_{\epsilon}\right)\right)^{-} \geq 0
$$

$$
\left(\lambda w_{\epsilon}\left(x_{\epsilon}\right)-H^{-}\left(x, D \phi\left(x_{\epsilon}\right)\right)\right)\left(w_{\epsilon}\left(x_{\epsilon}\right)-\psi_{1}\left(x_{\epsilon}\right)\right)^{-} \leq 0 .
$$

Now letting $\epsilon \rightarrow 0$, we get

$$
\left(\lambda v^{+}(\bar{x})-H^{-}(\bar{x}, D \phi(\bar{x}))\right)\left(v^{+}(\bar{x})-\psi_{1}(\bar{x})\right)^{-} \leq 0 .
$$

This proves that $v_{+}$is viscosity subsolution of the variational inequality (2.2). Similarly we can prove $v_{-}$is a viscosity supersolution of the variational inequality (2.2). Now using comparison principle (see Corollary 2.7), we have $v^{+} \leq v^{-}$. Also by definition we have $v_{-} \leq v^{+}$. Thus $v^{+} \equiv v_{-}$and $v$ is viscosity solution to (2.2). Uniqueness follows from the comparison principle.

We now show that the viscosity solution $v(\cdot)$ of the variational inequality $(2.2)$ is indeed the value function of the differential game of mixed type. We establish this result in the following theorem.

Theorem 3.5. The unique viscosity solution $v$ in $\mathcal{C}_{b}\left(\mathbb{R}^{d}\right)$ of the variational inequality (2.2) is the lower value function of the differential game of mixed type.

Proof. Using DPP, we have

$$
\begin{aligned}
w_{\epsilon}(x)= & \inf _{\alpha \in \Gamma} \sup _{u_{2} \in \mathcal{A}_{2}}\left[\int _ { 0 } ^ { \sigma } e ^ { - \lambda t } \left\{r\left(x(t), \alpha\left[u_{2}\right](t), u_{2}(t)\right)-\frac{1}{\epsilon}\left(w_{\epsilon}(x(t))-\psi_{1}(x(t))\right)^{+}\right.\right. \\
& \left.\left.+\frac{1}{\epsilon}\left(w_{\epsilon}(x(t))-\psi_{2}(x(t))\right)^{-}\right\} d t+e^{-\lambda \sigma} w_{\epsilon}(x(\sigma))\right]
\end{aligned}
$$


for any $\sigma \geq 0$. Hence

$$
\begin{aligned}
w_{\epsilon}(x) \geq & \inf _{\alpha \in \Gamma} \sup _{u_{2} \in \mathcal{A}_{2}}\left[\int _ { 0 } ^ { \sigma } e ^ { - \lambda t } \left\{r\left(x(t), \alpha\left[u_{2}\right](t), u_{2}(t)\right)\right.\right. \\
& \left.-\frac{1}{\epsilon}\left(w_{\epsilon}(x(t))-\psi_{1}(x(t))\right)^{+}\right\} d t \\
& \left.+e^{-\lambda \sigma} w_{\epsilon}(x(\sigma))\right] .
\end{aligned}
$$

Let $\theta_{m}=\inf \left\{t \geq 0: v(x(t))+1 / m \geq \psi_{1}(x(t))\right\}$. Then for $\sigma=\theta_{m} \wedge \tau$ for $\tau \geq 0$, we obtain

$$
\begin{aligned}
\int_{0}^{\theta_{m} \wedge \tau} e^{-\lambda t}\left(w_{\epsilon}(x(t))-\psi_{1}(x(t))\right)^{+} d t & \leq \int_{0}^{\theta_{m} \wedge \tau} e^{-\lambda t}\left(w_{\epsilon}(x(t))\right. \\
& \left.-\left(v(x(t))+\frac{1}{m}\right)\right)^{+} d t \\
& \leq\left(\int_{0}^{\theta_{m} \wedge \tau} e^{-\lambda t} d t\right)\left(\left\|w_{\epsilon}-v\right\|_{\infty}-\frac{1}{m}\right)^{+}
\end{aligned}
$$

Since $w_{\epsilon} \rightarrow v$, for sufficiently small $\epsilon$, the term on RHS is zero. Therefore

$$
\begin{aligned}
w_{\epsilon}(x) \geq & \inf _{\alpha \in \Gamma} \sup _{u_{2} \in \mathcal{A}_{2}}\left[\int_{0}^{\theta_{m} \wedge \tau} e^{-\lambda t} r\left(x(t), \alpha\left[u_{2}\right](t), u_{2}(t)\right) d t\right. \\
& \left.+e^{-\lambda\left(\theta_{m} \wedge \tau\right)} w_{\epsilon}\left(x\left(\theta_{m} \wedge \tau\right)\right)\right]
\end{aligned}
$$

for sufficiently small $\epsilon$. Now letting $\epsilon \rightarrow 0$, we obtain

$$
\begin{aligned}
v(x) \geq & \inf _{\alpha \in \Gamma} \sup _{u_{2} \in \mathcal{A}_{2}}\left[\int_{0}^{\theta_{m} \wedge \tau} e^{-\lambda t} r\left(x(t), \alpha\left[u_{2}\right](t), u_{2}(t)\right) d t+e^{-\lambda\left(\theta_{m} \wedge \tau\right)} v\left(x\left(\theta_{m} \wedge \tau\right)\right)\right] \\
\geq & \inf _{\alpha \in \Gamma} \sup _{u_{2} \in \mathcal{A}_{2}}\left[\int_{0}^{\theta_{m} \wedge \tau} e^{-\lambda t} r\left(x(t), \alpha\left[u_{2}\right](t), u_{2}(t)\right) d t\right. \\
& \left.+e^{-\lambda\left(\theta_{m} \wedge \tau\right)}\left\{v\left(x\left(\theta_{m}\right)\right) \chi_{\left\{\theta_{m} \leq \tau\right\}}+\psi_{1}(x(\sigma)) \chi_{\left\{\tau<\theta_{m}\right\}}\right\}\right] \\
\geq & \inf _{\alpha \in \Gamma} \sup _{u_{2} \in \mathcal{A}_{2}}\left[\int_{0}^{\theta_{m} \wedge \tau} e^{-\lambda t} r\left(x(t), \alpha\left[u_{2}\right](t), u_{2}(t)\right) d t\right. \\
& +e^{-\lambda\left(\theta_{m} \wedge \tau\right)}\left\{\psi_{2}\left(x\left(\theta_{m}\right)\right) \chi_{\left\{\theta_{m} \leq \tau\right\}}+\psi_{1}(x(\tau)\} \chi_{\left\{\tau<\theta_{m}\right\}}\right]-\frac{1}{m} \\
= & \inf _{\alpha \in \Gamma} \sup _{u_{2} \in \mathcal{A}_{2}} R\left(x, \alpha\left[u_{2}\right](\cdot), \theta_{m}, u_{2}(\cdot), \tau\right)-\frac{1}{m} .
\end{aligned}
$$


Since $\tau$ is arbitrary,

$$
v(x) \geq \sup _{\tau} \inf _{\alpha \in \Gamma} \sup _{u_{2} \in \mathcal{A}_{2}} R\left(x, \alpha\left[u_{2}\right](\cdot), \theta_{m}, u_{2}(\cdot), \tau\right)-\frac{1}{m} .
$$

Hence

$$
v(x) \geq \inf _{\theta} \sup _{\tau} \inf _{\alpha \in \Gamma} \sup _{u_{2} \in \mathcal{A}_{2}} R\left(x, \alpha\left[u_{2}\right](\cdot), \theta, u_{2}(\cdot), \tau\right)-\frac{1}{m} .
$$

Letting $m \rightarrow \infty$, we obtain $v(x) \geq V^{-}(x)$. Similarly we can prove the other inequality. This completes the proof of the theorem.

We now state the corresponding result for the upper value of the differential game of mixed type.

Theorem 3.6. The unique viscosity solution $v$ of the variational inequality (2.1) is the upper value function of the differential game of mixed type.

Combining Theorems 3.5 and 3.6, we obtain our main theorem.

Theorem 3.7. Under Isaacs minimax condition (2.10), the differential game of mixed type has value, i.e., $V^{+} \equiv V^{-}$and the value is the unique viscosity solution in $\mathcal{C}_{b}\left(\mathbb{R}^{d}\right)$ of both the HJI variational inequalities (2.1) and (2.2).

\section{Existence of a saddle point}

The aim of this section is to prove the saddle point equilibrium for the differential game described in first section when there is no control i.e., differential game with stopping times alone.

We now assume $b, r$ are independent of $u_{1}, u_{2}$. Note that in this case, Isaacs minimax condition is satisfied vacuously. Thus value function $v$ exists and is the unique bounded continuous viscosity solution of (2.1) (and hence (2.2)). Let $x \in$ $\mathbb{R}^{d}$. Let the stopping times $\hat{\theta}, \hat{\tau}$ be defined by:

$$
\hat{\theta}(x)=\inf \left\{s \geq 0: v(x(s))=\psi_{2}(x(s))\right\}, \quad \hat{\tau}(x)=\inf \left\{s \geq 0: v(x(s))=\psi_{1}(x(s))\right\},
$$

where $x(\cdot)$ is a solution of

$$
\dot{x}(t)=b(x(t)), \quad t>0, \quad x(0)=x .
$$

Then for each $t \in[0, \hat{\theta} \wedge \hat{\tau}), v(x(t)) \in\left(\psi_{2}(x(t)), \psi_{1}(x(t))\right)$. Let $C$ denote the set of all $x \in \mathbb{R}^{d}$ such that $\psi_{2}(x)<v(x)<\psi_{1}(x)$. Then $v$ is a viscosity solution of

$$
\lambda v(x)-b(x) \cdot D v(x)-r(x)=0
$$

in $C$. Then we have the following main theorem of this section.

Theorem 4.1. For each $x \in \mathbb{R}^{d},(\hat{\theta}, \hat{\tau})$ is a saddle point equilibrium. 
Proof. From the results of previous section, we have

$$
v(x)=\inf _{\theta \geq 0} \sup _{\tau \geq 0} R(x, \theta, \tau)=\sup _{\tau \geq 0} \inf _{\theta \geq 0} R(x, \theta, \tau) .
$$

We now show that

$$
v(x)=R(x, \hat{\theta}, \hat{\tau}) .
$$

If $x \notin C$, we have either $v(x)=\psi_{1}(x)$ or $v(x)=\psi_{2}(x)$. Then either $\hat{\theta}=0$ or $\hat{\tau}=0$. It is obvious to note that in either case $v(x)=R(x, \hat{\theta}, \hat{\tau})$. We now assume $x \in C$. Now applying the Proposition 5.18 and Remark, Chapter II, [1] to (4.1), we get for any $0 \leq \theta<\hat{\theta} \wedge \hat{\tau}$,

$$
v(x)=e^{-\lambda \theta} v(x(\theta))+\int_{0}^{\theta} r(x(t)) d t .
$$

Now letting $\theta \rightarrow \hat{\theta} \wedge \hat{\tau}$, we obtain

$$
v(x)=R(x, \hat{\theta}, \hat{\tau}) .
$$

Thus $(\hat{\theta}, \hat{\tau})$ constitutes a saddle point equilibrium.

\section{Conclusions}

In this paper we have studied a differential game problem of mixed type where each player uses both control and stopping times. By transforming this differential game problem of mixed type to a differential game with control only, we have shown that the variational inequalities associated with the differential game problem of mixed type have a unique viscosity solution. We then proceeded to prove the existence of value of differential game of mixed type via the penalization arguments. In Section 4, we have treated a special case of a differential game with only stopping times. Here we have established the existence of a saddle point equilibrium. This problem has been studied by Stettner in [6] for a class of Markov processes using the semigroup formulation. Thus the result for this special case in Section 4 is subsumed by the corresponding results in [6]. We have, however, used the method of viscosity solutions to arrive at the same result.

\section{References}

[1] M. Bardi and I. Capuzzo Dolcetta, Optimal Control and Viscosity Solutions of Hamilton-Jacobi-Bellman Equations, Birkhäuser, 1997.

[2] A. Bensoussan and J. L. Lions, Applications of Variational Inequalities in Stochastic Control, North Holland, 1982.

[3] L. C. Evans and P. E. Souganidis, Differetial games and representation formulas for soltions of Hamilton-Jacobi-Isaacs equations, Indiana Univ. Math. J. 33 (1984), $773-797$

[4] K. Fan, Fixed point and minimax theorems in locally convex topological linear spaces, Proc. Natl. Acad. Sci. 38 (1952), 121-126. 
[5] A. Friedman, Stochastic Differemtial Equations, Vol. 2, Academic Press, 1976.

[6] L. Stettner, Zero-sum Markov games with stopping and impulsive strategies, Appl. Math. Optim. 9 (1982), 1-24.

[7] J. Yong, A zero-sum differential game in a finite duration with switching strategies, SIAM J. Control Optim. 28 (1990), 1234-1250.

[8] J. Yong, Zero-sum differential games involving impulse controls, Appl. Math. Optim. 29 (1994), 243-261.

Mrinal K. Ghosh

Department of Mathematics

Indian Institute of Science

Bangalore 560012

India

e-mail: mkg@math.iisc.ernet.in

Mallikarjuna K. S. Rao

Industrial Engineering and Operations Research

Indian Institute of Technology

Mumbai 400076

India

e-mail: mallik.rao@iitb.ac.in

Dharmatti Sheetal

Laboratoire MIP

Université Paul Sabatier

31062 Toulouse Cedex 9

France

e-mail: sheetal@mip.ups-tlse.fr

Received: 31 July 2006.

Accepted: 10 July 2007. 